\title{
Fantasmes du millénaire : le futur du « genre » au $\mathrm{XXI}^{\mathrm{e}}$ siècle
}

Millenial Fantasies: The Future of "Gender" in the $21^{\text {st }}$ Century

\section{Joan Wallach Scott}

Traducteur : Myriam Boussahba-Bravard

\section{(2) OpenEdition \\ Journals}

\section{Édition électronique}

URL : https://journals.openedition.org/clio/9840

DOI : $10.4000 /$ clio.9840

ISSN : $1777-5299$

\section{Éditeur}

Belin

Édition imprimée

Date de publication : 31 décembre 2010

Pagination : 89-117

ISBN : 978-2-8107-0098-1

ISSN : $1252-7017$

\section{Référence électronique}

Joan Wallach Scott, «Fantasmes du millénaire : le futur du « genre » au xxı e siècle », Clio. Femmes,

Genre, Histoire [En ligne], 32 | 2010, mis en ligne le 31 décembre 2012, consulté le 28 avril 2022. URL : http://journals.openedition.org/clio/9840 ; DOI : https://doi.org/10.4000/clio.9840 


\title{
Fantasmes du millénaire : le futur du « genre » au XXI" siècle
}

\author{
Joan Wallach SCOTT ${ }^{*}$
}

Dans "Millenial Fantasies. The Future of "Gender" in the $21^{\text {st }}$ Century », un article publié en 2001 dans les Actes d'un colloque tenu en 1999 à l'Université de Berne en son honneur', Joan Scott développe une idée qu'elle avait déjà exprimée aux États-Unis lors de la réédition de Gender and the Politics of History ${ }^{2}$. Tandis qu'en Europe le terme de genre entre enfin de manière décisive dans le répertoire de la recherche académique, Joan Scott - dont l'article de 1986 constitue la référence à toute tentative de définition du genrẻ - le

* Je remercie Mary Louis Roberts, Debra Keates et Elizabeth Weed pour leur relecture attentive ainsi que Carol Lasser, Sandy Zagarell, Rosi Braidotti et Tony Scott pour leurs suggestions toujours constructives.

1 Joan W. SCOTT, "Millenial Fantasies. The Future of 'Gender' in the $21^{\text {st }}$ Century ", in C. Honegger \& C. ARni (eds), Gender. Die Tücken einer Kategorie. Joan Scott, Geschichte und Politik, Beiträge zum Symposion anlässlich der Verleibung des Hans-Sigrist-Preises 1999 der Universität Bern an Joan W. Scott, Zürich, Chronos Verlag, 2001, p. 19-37. Ce chapeau a été rédigé par Violaine Sebillotte Cuchet.

2 Joan W. SCOTT, Gender and the Politics of History [1988, 1re éd.], New York, Colombia University Press, édition révisée, 1999, avec une nouvelle préface et un nouveau chapitre : «Some more reflections on Gender and Politics », p. 199-222. Certains des articles de Joan Scott ont été récemment traduits par Claude ServanSchreiber et publiés dans J.W. Scott, Théorie critique de l'bistoire. I. Identités, expériences, politiques, Paris, Fayard, 2009. Un second volume paraitra en 2012 sous le titre Le Genre, une catégorie utile.

3 J.W. SCOTT, "Gender: a useful category of historical analysis », American Historical Review, 91/5, 1986, p. 1053-1075. Traduction française par E. Varikas sous le titre «Le genre : une catégorie utile de l'analyse historique », Les Cabiers du Grif, 37-38 1988, p. 125-155. 
déclare dépassé et contre-productif. On mesure alors le décalage qui sépare les études américaines et les études européennes non-anglophones : le genre, parce qu'il est devenu d'emploi banal dans le vocabulaire des organisations internationales, de même que dans des recherches américaines dorénavant dominées par les approches de la psycho-socio-biologie, a été détourné de l'emploi critique revendiqué dans les années 1980 par l'historienne féministe. Au-delà du mot, c'est la critique des savoirs et des usages «routiniers » qui est en jent.

Au début du $\mathrm{XX}^{\mathrm{e}}$ siècle, des voix prophétiques ont exprimé leur inquiétude pour l'avenir, notamment à propos des relations entre les sexes. Certains ont eu peur que les hiérarchies conventionnelles ne s'inversent : « la femme dominante » sortirait de son foyer sanctuarisé pour commander les armées, devenir avocate, ou présider le Parlement, laissant aux hommes le soin des enfants et le raccommodage. D'autres, plus optimistes ou peut-être plus désespérés à l'approche de la guerre, se sont représenté l'avènement du principe féminin dans un monde de coopération, d'harmonie et de paix perpétuelle. D'autres encore ont imaginé un monde sans sexe, dans lequel la reproduction serait scientifiquement organisée et où le plaisir deviendrait un acte entièrement cérébral. Alors, crurent-ils, l'égalité entre hommes et femmes serait possible. La variété de ces fantasmes nous rappelle qu'à chaque époque les contemporains ne partagent pas nécessairement les mêmes opinions. Qui plus est, intensément cloisonnés par nature, ces fantasmes contredisent notre expérience sociale et politique, qui est complexe, hasardeuse voire embrouillée. Ce seul fait devrait nous inciter à ne pas accorder trop de crédit aux prévisions millénaristes, qu'elles soient nôtres ou celles de nos contemporains. L'histoire échappe toujours aux explications structurelles que nous essayons d'imposer.

4 En 2007, une table-ronde réunissant à Paris Judith Butler, Éric Fassin et Joan Scott lui donne l'occasion de revenir sur les raisons de son inquiétude et d'exprimer son souhait d'un usage toujours critique du mot: «Pour ne pas en finir avec le 'genre'... », Sociétés et Représentations, 24, 2007, p. 285-306. 
Après avoir souligné la valeur prophétique limitée des fantasmes millénaristes, je dois néanmoins admettre que, personnellement, je suis la proie d'un scénario cauchemardesque dans lequel le déterminisme biologique revient pour contrôler le genre. Bien sûr, nous avons justement créé le terme genre pour combattre la pratique qui réduit les relations sociales à la différence anatomique des sexes. Il n'y a pas si longtemps, les féministes pensaient que «le genre » serait un rempart invulnérable face au déterminisme biologique. La distinction analytique sexe/genre séparerait le corps physique du corps social; il ne serait alors plus concevable que l'anatomie soit un destin. Lorsque l'anthropologue Gayle Rubin écrit son article bien connu sur le système sexe/genre, elle s'autorise même à rêver à l'élimination de la sexualité obligatoire et des rôles sexués.

Mon plus beau rêve est celui d'une société androgyne sans genre (mais non pas sans sexe) dans laquelle l'anatomie sexuelle ne déterminerait pas qui on est, ni ce qu'on fait, ni avec qui on fait l'amour ${ }^{5}$.

Dans mon cauchemar, «le genre» se révèle être la ligne Maginot du féminisme, impuissant à arrêter le retour du raisonnement qui conduisit le biologiste écossais Patrick Geddes à alerter les suffragistes des années 1880 sur la futilité de leurs revendications. Geddes maintenait que deux formes de métabolisme cellulaire expliquaient la différence entre les sexes : «la cellule active et affamée devient un spermatozoïde énergique tandis que la cellule rassasiée devient tranquillement un ovule ». Il s'ensuit que «ce qui fut décidé parmi les protozoaires préhistoriques ne peut pas être annulé par une loi parlementaire $\aleph^{6}$. Bien que l'on ne conteste plus aux femmes le droit de voter, ni d'ailleurs celui de participer à la vie publique et professionnelle, il existe aujourd'hui un courant puissant parmi les scientifiques qui, comme dans le cas de Geddes, consiste à importer la théorie de l'évolution dans le champ social et politique. Connu sous le nom de sociobiologistes dans les années 1970, «les psychologues évolutionnistes » d'aujourd'hui concluent qu'il existe des différences universelles entre hommes et femmes; ils déduisent ces différentes

5 Rubin 1975.

6 Patrick Geddes \& J. Arthur Thompson, The Evolution of Sex, Londres, 1889, New York, 1890, cité in Conway 1972: 146. 
caractéristiques comportementales et émotionnelles des fonctions reproductives. Bien sûr, aujourd'hui, la connaissance scientifique est bien plus sophistiquée qu'elle ne l'était à l'époque de Geddes; le gène a remplacé la cellule comme unité fondamentale de la transmission des caractères héréditaires. Cependant, l'argumentaire des psychologues évolutionnistes résonne comme un écho familier et dérangeant pour quiconque a lu les déclarations du XIXe siècle : les hommes chercheraient à disséminer leur semence pour assurer leur transmission génétique, disent ces psychologues, tandis que les femmes seraient plus sélectives dans le choix de leurs partenaires; elles miseraient sur la sociabilité et l'entraide pour mieux investir leur temps et leur énergie dans la grossesse, puis dans l'éducation des enfants. Puisque, affirment-ils encore, ces adaptations évolutionnistes garantissent la survie humaine, ils en déduisent « la manière essentielle dont chacun ressent des émotions envers autrui, ce qu'il pense des autres et ce qu'il leur dit $[\ldots] »^{7}$. J'ai entendu des psychologues évolutionnistes appliquer ce raisonnement fondé sur la reproduction au comportement d'enfants de cinq ans en récréation (les garçons se battent, les filles s'associent) ainsi qu'au débat intellectuel entre étudiants (les jeunes hommes se mettent en valeur, les jeunes femmes se regroupent). Le New York Times a récemment publié un article sur la recherche de deux scientifiques qui déclarent que le viol n'a rien à voir avec la violence et le pouvoir, mais tout avec la procréation car il « exprime» la faculté d'adaptation des hommes ${ }^{8}$. Un ouvrage récent défend sa propre lecture évolutionniste des femmes au travail en attribuant le plafond de verre et autres injustices actuelles liées au genre « aux différences de développement entre les sexes »9. Certaines se proclamant féministes célèbrent le potentiel politique, parait-il caractéristique, des femmes: leur capacité relationnelle ou leur sociabilité serait génétiquement fondée ${ }^{10}$. Même un critique de la

7 Robert Wright, The Moral Animal, New York, Pantheon Books, 1994, cité in Gould $1997: 151$.

8 Erica Goode, "What Provokes a Rapist to Rape?", The New York Times, 15 janvier 2000, p. 9, 11.

9 Publicité pour Browne 1999.

10 Un exemple récent est Fisher 1999. 
psychologie évolutionniste aussi virulent que Stephen J. Gould reconnait que «les exigences darwiniennes demandent aux hommes et aux femmes un comportement distinct pour s'adapter» qui «probablement sous-tend certaines tendances émotionnelles quasi générales chez les humains mâles et femelles ${ }^{11}$, alors même qu'il est un adversaire farouche de telles idées appliquées à l'ensemble des activités sociales. Encore plus révélateur pour moi a été le numéro spécial de la revue History and Theory sur «Le retour de la science : les idées évolutionnistes et l'histoire ». La plupart des huit articles s'enthousiasment pour un mariage de la biologie et de l'histoire (ce qui rappelle l'engouement pour l'histoire quantitative des années 1970) visant non seulement « une science de la nature humaine » mais aussi «l'unité de la connaissance », rêve fou du démiurge scientifique. Avec une expression que je laisse le soin aux psychanalystes d'interpréter, le responsable du numéro note que «la science force l'histoire $\gg^{12}$. Un des contributeurs conclut son article avec une phrase tout à fait surprenante, mélange de souhait et de prophétie : "Introduire l'évolution darwinienne dans les cursus d'histoire serait une modification paradigmatique d'une magnitude copernicienne $»^{13}$. Pendant la rédaction de cet essai, j’ai reçu un courriel d'un collègue qui travaillait auparavant sur l'histoire sociale et politique américaine ; il m’invitait ainsi que E.O. Wilson à une table ronde organisée sur le thème de la «bio-histoire » par l'American Historical Association. Parmi les questions à débattre figuraient «Pourquoi les historiens ont-ils si peur de Darwin ? », "Si l'histoire est entièrement 'construite', quels en sont les matériaux de construction ?», ainsi que celle-ci ajoutée pour moi, j'en suis sûre, «Reconnaître le poids de l'inné dans le débat sur l'histoire signifie-t-il que les femmes sont considérées comme inférieures?».

Celles d'entre nous qui pensaient que « le genre » avait contribué à la défaite de la sociobiologie, qui traduit directement la différence anatomique en comportement social, celles d'entre nous qui

\footnotetext{
11 Cité in Schwartz 1999: 20.

12 Shaw 1999 : 9.

13 Dawson $1999: 100$.
} 
pensaient que l'usage généralisé du terme genre indiquait combien notre position avait progressé, sont abasourdies de voir dans le succès actuel de la psychologie évolutionniste le retour triomphal de la sociobiologie. Proche des théories évolutionnistes qui sous-tendent la biologie moléculaire et neurologique, sciences qui, nous dit-on, vont remplacer au $\mathrm{XXI}^{\mathrm{e}}$ siècle le règne hégémonique des sciences physiques, la psychologie évolutionniste occupe maintenant une position de force pour annuler, si ce n'est inverser, cent années de critique féministe.

Comme toutes les prévisions millénaristes (et comme beaucoup de fantasmes), cette dernière affirmation est hyperbolique. Elle réduit considérablement un champ traversé par de multiples courants à une lutte manichéenne entre le féminisme et le néo-darwinisme pour déterminer le sens futur de la différence sexuelle. En outre, elle rejette les développements dus au hasard et à la contingence qui influencent à la fois les sciences naturelles et l'histoire. Pourtant, réfléchir ainsi est utile; cela permet d'identifier les questions qu'il faut aujourd'hui considérer et rapidement trancher. En effet, il y a urgence. Nous, féministes, devons pouvoir nous mobiliser, redoubler d'efforts contre le déterminisme biologique; nous devons réévaluer la manière dont nous le rejetons. C'est cette deuxième voie que je veux explorer aujourd'hui. $\mathrm{Si}$, dans une veine freudienne, un cauchemar peut exprimer un souhait (trop horrible ou trop difficile à reconnaittre), de même, (malgré un consensus féministe assez répandu, d'où la difficulté de ce que je souhaite défendre), on pourrait conclure que le genre n'est plus la catégorie utile qu'il a été, non pas parce que l'ennemi a gagné, mais parce qu'utiliser ce terme aujourd'hui ne fait plus avancer la cause féministe.

Aujourd'hui le genre est incapable de combattre les prétentions extrêmes de la psychologie évolutionniste pour la raison même qui fut à l'origine de son succès : son refus de considérer le sexe corporel. Empruntée par les féministes des années 1960 aux endocrinologues et psychanalystes (principalement à John Money et à Robert Stoller), la distinction sexe/genre insistait sur le fait que les rôles sexuels étaient une invention humaine, qu'ils étaient naturalisés en référence au corps physique sans que celui-ci ne les détermine. Le but était d'étudier la façon dont «la construction sociale» se produisait, de 
démontrer sa variété et sa mutabilité, de révéler son mode opératoire comme système de pouvoir et de proposer des alternatives ou des résistances à cette prescription normative. Cette démarche a produit énormément de travaux dont je ne veux pas méconnaître ou sousestimer l'importance. Par ailleurs, je ne veux pas non plus m'associer aux idées de Camille Paglia ou de certains théoriciens queer qui affirment que s'occuper du genre en négligeant le sexe constitue une forme de puritanisme féministe ${ }^{14}$. En revanche, plus encore qu'avant, je veux apporter mon soutien total au dit "féminisme français", position certes minoritaire parmi les féministes américaines, mais qui interroge l'usage social et scientifique du "genre» au nom de la psychanalyse. Ces féministes, qui veulent à la fois insister sur l'indétermination des positions du sujet sexué et faire valoir les inégalités de pouvoir, préfèrent parler de la différence sexuelle et des « rapports de force de sexe » (NdTR français original).

La distinction sexe/genre telle qu'elle a été développée par la majorité des féministes américaines laisse de côté, et donc en place, le corps sur lequel on érige ces constructions comme s'il appartenait à la nature et donc était non interrogeable. Par conséquent, le sexe a continué à miner la clarté que «le genre» était censé apporter. Voyons le commentaire sur l'usage du mot "genre» dans The American Heritage Dictionary of the English Language (1992) :

Traditionnellement, on utilise le genre principalement pour décrire les catégories grammaticales du «masculin », «féminin » et " neutre »; mais, récemment, l'usage a solidement établi ce mot comme la référence à des catégories fondées sur la différence des sexes dans des expressions comme linégalité de genre ou la politique de genre. Cet usage correspond à la pratique de nombreux anthropologues qui réservent sexe aux catégories biologiques, alors qu'ils utilisent genre pour se référer aux catégories sociales et culturelles. Selon cette règle, on dira L'efficacité de ce médicament semble dépendre du sexe (non pas du genre) du patient, mais Dans les sociétés paysannes, les rôles liés au genre (non pas au sexe) ont tendance à être plus clairement définis. Utile en principe, cette distinction n'est en aucune façon

14 NdE. Auteure entre autres de Sex, Art and American Culture: Essays (1992), C. Paglia a provoqué de nombreuses controverses parmi les féministes américaines. 
courante et comporte des variations considérables d'usage à tous les niveaux ${ }^{15}$.

Cette dernière phrase est cruciale parce qu'elle nous rappelle à la fois combien il est futile d'insister sur un usage linguistique précis et combien il a été difficile pour les féministes de séparer la signification sociale de son référent physique. Malgré toute l'insistance des théoriciennes féministes à expliquer le terme genre, elles n'ont pas pu empêcher la corruption de son emploi. Dans la conversation ordinaire, on emploie autant les termes de sexe et de genre comme synonymes que comme contraires. Le genre est parfois devenu, semble-t-il, un euphémisme poli pour le sexe. Et si l'on en croit le nombre de livres et d'articles savants qui considèrent que le genre et les femmes sont des synonymes, les universitaires ne sont pas plus doués que le grand public pour faire la distinction entre le physique et le social, objectif initial du terme genre.

Je pense que la confusion de ces deux termes est symptomatique d'un certain nombre de problèmes connexes qui viennent tous du fait que l'opposition sexe/genre reproduit l'opposition nature/culture et l'opposition corps/esprit. À chaque fois, on considère «la nature » comme une entité extérieure à la réflexion humaine, que l'on doit apprendre à connaître alors qu'elle n'est pas supposée appartenir à la connaissance. Si le genre est l'usage que nous faisons de notre corps, ce dernier ne peut être compris entièrement en termes de construction sociale. Il ne remplace donc pas le sexe physique dans le débat sur la différence sexuelle; mais, au bout du compte, le genre laisse le sexe en place comme explication de la construction sociale. Quand le genre dépend ainsi du sexe, rien ne peut l'empêcher d'être confondu avec lui, ou compris en tant que tel. Ce qui semble alors être une confusion de conception et de terminologie est, en fait, une représentation exacte de l'interdépendance des deux termes : si le sexe n'appartient pas entièrement à la nature, le genre n'appartient pas non plus entièrement à la culture.

Une autre raison nous empêche de clairement distinguer le sexe du genre ; c'est l'universalisme commun au féminisme (mouvement

15 The American Heritage Dictionary of the English Language, Boston, Houghton Mifflin, 1992 (3e éd.), p. 754. 
politique qui naît en Occident au moment des révolutions démocratiques du XVIII ${ }^{e}$ siècle) et aux sciences sociales (dont les origines sont approximativement contemporaines du féminisme). Leur tendance universaliste a contribué à produire une vision des femmes fondamentalement homogène (au-delà des époques et des cultures) en considérant que la différence essentielle entre les femmes et les hommes allait de soi. Même prises en compte, les différences nationales et/ou culturelles sont traitées comme des phénomènes secondaires, comme autant de variations d'un thème universel pour lequel le genre signifie toujours la même chose: une relation asymétrique, si ce n'est antagoniste, entre les femmes et les hommes, qui organise les fonctions de chacun en activités et espaces séparés. Mais si le genre, invariant de la différence sexuelle, est universel, comment, finalement, expliquer son universalité en dehors de la biologie? Si le genre signifie les formes sociales plaquées sur la différence physique existante entre les femmes et les hommes, alors la nature (que ce soit le corps ou le sexe) reste en place comme facteur déterminant de la différence. Si l'étude des femmes mène automatiquement à "l'analyse du genre», alors une forme d'essentialisme dirige la recherche : la présence de femmes, anatomiquement parlant, signifie alors qu'un système de différence opère, système que nous connaissons déjà. Lorsque «le genre » présuppose des différences sexuelles physiques, quand il en devient de fait un synonyme, alors maintenir une distinction claire et conceptuelle entre le sexe et le genre s'avère difficile. En outre, l'historicisation que le genre était supposé opérer vis-à-vis du sexe ne peut plus être effectuée puisque la biologie est considérée comme anhistorique.

La fixité de l'opposition masculin/féminin, son absence d'histoire, est, bien sûr, un axiome des psychologues évolutionnistes. Alors que le mot «évolution » devrait impliquer des changements au cours du temps, en fait, tout dans leur discours distingue «évolution» et «histoire », la première se référant au "processus des lois de la sélection et de l'adaptation », l'autre à la contingence, à la spécificité 
contextuelle et à l'historicité16 (c'est ce que montrent Richard Lewontin et Joseph Fracchia dans le numéro de History and Theory dont j'ai parlé précédemment). Les psychologues évolutionnistes posent le principe d'un moment originel où le temps s'arrête pour les espèces. Ainsi Steve Pinker, psychologue au Massachusetts Institute of Technology écrit (lorsqu'il défend le Président Clinton lors du scandale Monica Lewinsky) :

La plupart des pulsions humaines fonctionnent sur d'anciens postulats darwiniens $[\ldots]$ Un homme préhistorique qui couchait avec cinquante femmes pouvait engendrer cinquante enfants, et il aurait eu plus de chances d'avoir des descendants qui hériteraient de ses goûts. Une femme qui couchait avec cinquante hommes n'aurait pas eu plus de descendants qu'une femme qui couchait avec un seul homme. Donc, les hommes devraient [remarquez comment le temps du verbe passe du conditionnel passé au futur de recommandation] rechercher la quantité, les femmes la qualité dans leurs partenaires sexuels ${ }^{17}$.

Avec une précision pseudo-scientifique, ce scénario fantasmatique fixe dans la préhistoire la réalité de caractéristiques anciennes, mais ne prend pas en compte les siècles de changement dans notre environnement ni les stratégies d'adaptation qui ont été nécessaires depuis. Incidemment, il est ironique (ou, au contraire, peut-être prévisible) que cette insistance à lier le comportement social/sexuel des hommes aux impératifs de la reproduction arrive à un moment où les nouvelles technologies menacent d'éliminer les hommes, mais non leur sperme, de la procréation.

Les arguments de « la construction culturelle» du genre n'ont pas réussi à combattre efficacement cette théorie de l'évolution qui détache l'activité humaine de tout contexte temporel, pour au moins

16 «Les différences entre ces deux perspectives sont incommensurables, non pas à cause des frontières disciplinaires, mais parce qu'elles impliquent des conceptions différentes sur la nature de l'enquête 'scientifique', des postulats ontologiques et épistémologiques différents et donc des modes d'explications différents", Fracchia \& Lewontin 1999 : 52-58, citation 58. Pour une critique plus développée des fondements de la psychologie évolutionniste voir Lewontin 1984 ; Dupré 1993a, 1993 b.

17 Pinker 1988. Pour une critique de Pinker, voir Dupré 1999: 489-493. Voir également Malik 1998 ; Deacon 1997. 
deux raisons ${ }^{18}$. J'ai déjà fait allusion à la première; alors qu'on a donné une histoire au genre, le sexe biologique n'en a toujours pas. La seconde raison est en rapport avec la première; nous les féministes avons utilisé la théorie de la construction culturelle pour récuser la légitimité de la science; et ceci nous a souvent empêchées de nous occuper de certains aspects du savoir biologique qui auraient pu utilement historiciser le sexe anatomique. Nous avons analysé la politique du discours scientifique qui légitime les inégalités sociales entre les femmes et les hommes; nous avons insisté sur les insuffisances empiriques de certaines descriptions catégorielles des femmes. Mais (à l'exception de scientifiques comme Anne Fausto Sterling et Donna Haraway) nous ne nous sommes pas confrontées à l'autorité épistémique de la biologie elle-même ${ }^{19}$. Il y a une douzaine d'années, Donna Haraway remarquait que s'efforcer « de détacher les femmes de la catégorie de la nature pour les définir comme sujets sociaux se construisant dans la culture, mettait le concept de genre en quarantaine contre les infections du sexe biologique $»^{20}$. Insister sur la «construction» de la science (qui énonce comme postulat sa propre objectivité ainsi que la transparence de la nature) a certes remis en cause son autorité, mais a empêché un engagement sur son propre terrain. À la liste d'oppositions sexe/genre, nature/culture, corps/esprit s'est ajoutée celle de science/féminisme. Toutes ces associations inversent l'opposition habituelle masculin/féminin qui pose le principe de l'esprit et du masculin contre le sexe et le féminin, car, finalement, l'opposition science/féminisme accorde le sexe à la science et place les femmes en dehors de celle-ci ${ }^{21}$. Nous, les féministes, pouvons alors dénoncer la partialité politique de la psychologie évolutionniste qui défend vigoureusement le fondement biologique du genre; cependant, nous sommes impuissantes à

18 La critique de la théorie évolutionniste faite par Clifford Geertz au nom des spécificités culturelles n'a pas influencé beaucoup les travaux développés sous l'égide du genre, à l'exception de quelques anthropologues et historiens des sciences. Voir Geertz 1973 : chapitres 2, 3. Voir également Rosaldo 1980.

19 Sterling 1985, 2000 ; Haraway 1991.

20 Haraway, "'Gender' for a Marxist Dictionary: the Sexual Politics of a Word », in Haraway 1991 : 134.

21 Wilson 1998. 
contester concrètement la génétique évolutionniste qui est la science qui le démontre, et nous sommes impuissantes à trouver les généticiens et généticiennes qui pourraient articuler une position différente dans leur recherche. Ainsi, nous abandonnons aux psychologues évolutionnistes le terrain qui est notre cible; nous permettons alors à des modèles (voire des fantasmes) de savoir scientifique d'apparaitre comme cohérents et unifiés et donc d'être seuls représentants d'un champ qui, dans les faits, est contestable et contesté22.

La science est une forme de savoir dont l'organisation aussi a une histoire; ce n'est pas une histoire politique au sens étroit, celle qui traiterait la science comme un simple reflet des préjugés sociaux de son époque, mais une histoire conflictuelle des concepts et des principes organisationnels par lesquels nous percevons la nature. (Au sein de la biologie, par exemple, il y a des différences majeures, «des guerres civiles ", m'a-t-on dit, sur le statut de l'explication scientifique $)^{23}$. Ainsi, il se pourrait que l'amalgame du sexe et du genre dans l'usage courant soit une correction de «l'erreur» qui traite le sexe et la nature comme des entités transparentes situées en dehors de la « culture ». En lieu et place, on doit donc comprendre le genre et le sexe comme des systèmes de savoir interdépendants dont les rapports sont complexes. Bien sûr, le succès de l'opposition sexe/genre a été précisément de traiter le sexe anatomique comme une forme de savoir social, mais elle a aussi trop insisté sur sa détermination sociale externe, négligeant ainsi l'histoire des idées scientifiques en ce qu'elles ont d'autonome (par exemple, la génétique ne peut pas être réduite à un reflet de la lutte des classes ou des sexes dans le capitalisme actuel). J'approuve la neuropsychologue Elizabeth Wilson quand elle écrit que l'attention particulière portée au genre et la définition étroite de leur objet d'études - les femmes - a écarté les féministes du débat avec les sciences de la vie. Je suis aussi d'accord avec son constat, que «le féminisme doit combattre l'autorité scientifique pas simplement dans les lieux où elle prend les femmes

22 Sur la façon dont les fantasmes imposent une certaine cohérence aux phénomènes chaotiques, voir ̌̌ižek, 1997.

23 Fracchia \& Lewontin 1999: 59. 
pour objets, mais aussi dans les zones neutres, celles dans lesquelles le féminisme semble n'avoir ni place, ni intérêt politique». Pour E. Wilson, ceci signifie développer une recherche non pas sur les différences de capacités cognitives selon le sexe, mais sur la «nature de la cognition elle-même $»^{24}$. Pour traiter le problème que pose la psychologie évolutionniste, l'approche d'Elizabeth Wilson s'attacherait, je pense, moins à questionner la priorité que les femmes donnent au relationnel, qu'à analyser la manière dont leurs corps enregistrent leurs histoires. Le genre n'est pas une catégorie particulièrement utile pour penser en ces termes-là.

Dans la psychologie «connective », E. Wilson trouve des théories dynamiques de la cognition qui remettent en question les notions simplistes du déterminisme biologique. La fonction cérébrale et la conscience, affirme-t-elle, sont bien plus complexes que ce que la programmation génétique peut expliquer par des notions réductrices. Au lieu de cela, fait-elle remarquer, la recherche montre que les modèles cognitifs s'établissent différemment au cours de l'histoire individuelle. Dans une veine plus utopique, Elizabeth Grosz suggère que l'étude du corps pourrait fournir les munitions dont ont besoin les féministes :

[...] n'est-ce pas plus troublant de montrer non pas que le genre peut ne pas s'accorder avec le sexe [...], mais qu'il y a une instabilité au cœur du sexe et du corps, que le corps est ce qu'il est capable de faire, et que ce que chacun est capable de faire dépasse de loin les limites que chaque culture se fixe ?25. $^{25}$.

Si certaines entendent ici l'écho familier des dernières pages énigmatiques de l'Histoire de la sexualité (tome 1) de Foucault, je pense qu'elles ont raison. Celles qui voulaient combattre l'essentialisme au nom du genre se sont penchées avec inquiétude sur leur sens :

$\mathrm{Ne}$ pas croire qu'en disant oui au sexe, on dit non au pouvoir [c'était l'argument contre l'idée de la libération sexuelle; le livre avait montré que le sexe n'était pas une force naturelle en dehors de l'histoire, mais un effet des idées sur la sexualité] [...]. C'est de l'instance du sexe qu'il faut s'affranchir si, par un retournement tactique des divers mécanismes de la sexualité, on veut faire valoir contre les prises de pouvoir, les corps, les

\footnotetext{
24 Wilson 1998 : 18-19.

25 Grosz 1994 : 140.
} 
plaisirs, les savoirs, dans leur multiplicité et leur possibilité de résistance. Contre le dispositif de sexualité, le point d'appui de la contre-attaque ne doit pas être le sexe-désir, mais les corps et les plaisirs ${ }^{26}$.

Je comprends maintenant que Foucault ne faisait pas appel à un corps transparent hors conceptualisation, mais à une entité matérielle qu'E. Wilson juge «bio-culturelle ». «C'est le corps dont l'expérience repose sur un ensemble de paramètres bio-culturels et biopsychiques $»^{27}$. Pour E. Wilson, le but de la science féministe est de produire un savoir du corps qui interagit avec, mais aussi dépasse les possibilités du paramétrage physique dans lequel il opère. D’après son analyse, le corps est produit par des impressions contingentes (radicalement individualisées) qui combinent réactions sensorielles et fantasmes inconscients (neurologiquement enregistrés) de sorte que le déterminisme biologique comme la séparation esprit/corps deviennent des absurdités totales.

Il est intéressant, et probablement peu surprenant, de constater qu'E. Wilson relie son travail à la psychanalyse. Dans sa recherche en neurologie, elle utilise le fait que la psychanalyse tente d'historiciser le corps en refusant l'opposition esprit/corps (c'est pourquoi l'ouvrage de Freud Esquisse pour une psychologie scientifique est particulièrement important à ses yeux, tout autant que l'est la longue filiation de théorisation féministe associée à la psychanalyse et à la déconstruction). Il est possible que mon propre intérêt pour certaines théories de la psychanalyse soit une réponse perverse aux attaques récentes portées contre elle, mais il se trouve aussi que seule la psychanalyse interroge l'identité et le comportement sexuels de manière aussi directe. Comme E. Wilson, je trouve pertinent ces récits du développement humain qui comprennent la pulsion et le désir non pas comme des besoins innés, mais comme des produits d'histoires individuelles. Prenons par exemple la discussion de Freud dans Trois essais sur la théorie sexuelle sur la façon dont le nourrisson en vient à vivre la tétée non seulement comme un moyen d'apaiser sa faim, mais aussi comme une source de plaisir oral. Indépendamment du besoin de se nourrir, la bouche et les lèvres deviennent une zone

26 Foucault $1976: 207-208$.

27 Wilson 1998 : 64. 
érogène, moteurs du désir corporel qui précède la pensée consciente. Ces enfants qui au sein éprouvèrent tôt une grande satisfaction sexuelle, écrit Freud, peuvent plus tard «devenir des amateurs de baisers, [ou] rechercher les baisers pervers $[\ldots] »^{28}$. Ce point, souligné différemment et de façon plus complexe par Lacan, implique que les pulsions orales comme celles associées à la vue, l'ouie et aux parties génitales, ne sont pas purement biologiques. L'expérience physique dont on se souvient inconsciemment investit le corps d'une signification qui dépasse ce qui peut être expliqué par le besoin purement physique $^{29}$. Ce processus de formation et de développement (cette histoire individualisée) produit des désirs qui, de toute évidence, n'appartiennent pas au comportement jugé masculin ou féminin et qui ne peuvent pas être lus a posteriori comme une détermination génétique. Ce processus persiste lorsque les individus confrontent, s'adaptent et résistent aux règles et attentes sociales spécifiques à une époque. Les individus sexués (pourvus de pulsions, de désirs et de corps) qui vivent ce processus ne sont les produits exclusifs ni du « genre » ni de la biologie.

Pour Freud, le défi était d'analyser les interactions entre la variation individuelle et la catégorisation sociale que les psychologues évolutionnistes, eux, préfèrent ignorer :

[...] ni du point de vue biologique ni du point de vue psychologique, les caractères d'un des sexes chez un individu n'excluent ceux de l'autre. Tout être humain, en effet, présente au point de vue biologique, un mélange des caractères génitaux propres à son sexe et des caractères propres au sexe opposé, de même qu'un mélange d'éléments actifs et passifs, que ces éléments d'ordre psychique dépendent ou non des caractères biologiques ${ }^{30}$.

(Ceci n'est pas le récit freudien de la différence sexuelle, mais une tentative de problématiser ce qui est souvent compris comme des différences de nature entre les sexes) ${ }^{31}$. De plus, il n'y a aucune garantie que les enfants s'identifieront au parent de leur sexe physique dans leur parcours vers l'âge adulte. Au lieu de cela, un processus

\footnotetext{
28 Freud $1962: 75$.

29 Pour Lacan voir Miller 1978. Voir également Shepherdson 2000 : 85-113.

30 Freud $1962: 185$.

31 Merci à Debra Keates pour cette remarque.
} 
complexe d'identification imprègne chaque individu dans l'incorporation qu'il fait du masculin et du féminin. Ce processus résiste aussi aux corrélations faciles entre le sexe anatomique et le genre socialement construit comme Judith Butler le montre si bien en étudiant les identités sexuées grâce aux concepts psychanalytiques :

Que se passe-t-il, [demande-t-elle], lorsque les interdits primaires de l'inceste produisent des déplacements et des substitutions non conformes [aux modèles culturels de l'hétérosexualité] ? [...] Il se peut en effet qu'une femme trouve des reliquats fantasmatiques de son père dans une autre femme ou reporte sur un homme son désir pour sa mère $[. .$. [Si] un homme peut s'identifier à sa mère, et produire du désir à partir de cette identification [...], il brouille déjà la description psychique du développement de genre stable. Et si ce même homme désire un autre homme, ou une femme, son désir est-il homosexuel, hétérosexuel, voire lesbien? 32

Ces questions sur l'identité individuelle et le rôle de l'identification fantasmatique dans sa construction transforment les catégories "homme» et "femme» en des idéaux établis pour contrôler le comportement; ils ne correspondent pas à la description de gens réels, ce qui condamne ces derniers à échouer s'ils veulent les réaliser. En effet, ces catégories supposent qu'il est possible d'atteindre les idéaux normatifs de la masculinité et de la féminité, ce que les institutions politiques et sociales poussent à faire. Les termes d'«homme » et de "femme », comme tous les signifiants, ont donc des sens qui ne sont ni fixes ni absolus : ils s'établissent dans l'espace différentiel de la relation entre l'un et l'autre. Les historiens et anthropologues qui travaillent sur les identités collectives ont montré combien ces idéaux normatifs sont variables, combien, par exemple, les impératifs du mariage et de la reproduction dépendent d'idées sur, disons, l'importance de la parenté pour transmettre la propriété et le pouvoir, ou combien le nombre d'habitants traduit la force d'une société ou d'un État. Est-il besoin de dire que les notions de masculinité et de féminité diffèrent selon « le régime reproductif »33 ? En d'autres termes, le comportement reproductif tout comme le

32 Butler 2009 : 108-109.

33 Comme exemples de travaux anthropologiques et historiques, voir Ortner \& Whitehead 1991 ; Emilio \& Freedman 1988. 
corps reproducteur dépend du contexte; comme il se doit, les choses se compliquent, quand on ajoute que le corps se reproduit parfois sous la contrainte psychologique quand elle n'est pas physique.

Je ne veux pas reprendre ici l'ensemble des points soulevés par les déclarations réductrices de la psychologie évolutionniste. Mon objectif est de commencer à explorer les limites du genre comme catégorie d'analyse et la psychologie évolutionniste fournit une piste d'exploration. Cependant, j'étais déjà gênée par l'utilisation de ce terme avant de m'apercevoir du retour du biologisme. Le genre était devenu une routine qui contribuait à la stabilité de l'opposition homme/femme plutôt qu'il ne la remettait en question. J'en ai eu assez de me trouver citée en permanence dans des livres ou des articles qui tenaient pour acquis la transparence de la différence physique entre les sexes, qui élevait le genre au rang de théorie alors que, en fait, il servait de synonyme aux catégories non interrogées de «femmes» et $d^{\prime}$ '«hommes ». Mon utilisation personnelle du genre reposait, et c'est toujours le cas, sur qu'on a appelé le linguistic turn. J'ai été consternée de voir cette théorie évacuée par la thématisation du «genre».

Il est absolument certain que ce terme a été extrêmement utile. Dans les années 1970 et 1980, grande période de son appropriation par le féminisme, les limites du genre sont apparues aux yeux de certaines critiques (Donna Haraway aux États-Unis, Luce Irigaray en France, pour n'en citer que deux), mais le concept permettait encore d'accomplir d'importants travaux théoriques et politiques. Il nous a permis de séparer la biologie de la culture (distinction néfaste peutêtre à long terme, mais d'abord utile), d'être d'accord avec Simone de Beauvoir (contre les sciences sociales fonctionnalistes) qu'« on ne nait pas femme » et de justifier le changement dans les relations entre les sexes comme le produit d'un processus historique. De plus, le mot genre lui-même était discordant car il transposait un concept grammatical (même si c'était par le biais du discours médical) dans le champ de l'identité sociale et sexuelle des humains. Je ne sais combien d'universitaires (plus âgés) ont courtoisement exprimé leur gêne à m'entendre parler en flagrant délit de mauvais usage, pensaient-ils (avec un malin plaisir): «n'est-ce pas une référence grammaticale? », demandaient-ils poliment à la fin d'une 
intervention ${ }^{34}$. Cependant leur remarque me fournissait l'occasion d'expliquer ce que j'avais en tête et de remettre en question la vision prévalant dans le monde entier qui niait aux femmes (et aux relations entre hommes et femmes) une histoire. C'était également délicieusement canaille d'utiliser un terme grammatical pour parler de la différence sexuelle. En grammaire, le genre est considéré comme une manière de classifier les phénomènes: c'est un système de distinctions socialement sanctionnées plutôt qu'une description objective de traits inhérents. C'était exactement la raison pour laquelle nous utilisions le genre au lieu du terme sexe en débattant du rôle et du comportement des hommes et des femmes, qui n'étaient pas naturels mais prescrits et assignés (et puis, il y avait aussi la possibilité de remettre en question les présupposés hétérosexuels interrogés par le fait que dans certaines langues indo-européennes le genre inclut non pas deux, mais trois catégories : masculin, féminin et neutre).

En histoire et dans les sciences sociales en général, le genre a ouvert la voie à ce qu'on pourrait appeler l'étude sociale de la différence sexuelle. Nous demandions dans quelles conditions on avait défini les rôles et les fonctions différentes pour chaque sexe, comment on avait créé et imposé les normes régulant le comportement sexuel, comment les questions de pouvoir et de droits influençaient les définitions du masculin et du féminin, comment les structures symboliques affectaient la vie et les pratiques des gens ordinaires, comment les identités sexuelles se forgeaient dans, mais aussi contre les prescriptions sociales. Dans le contexte de l'épidémie de SIDA et grâce à l'intervention explosive des théoriciens queer, la position ancienne du féminisme psychanalytique s'est retrouvée sur le devant de la scène : l'hétérosexualité elle-même a été redéfinie comme un système normatif et non pas «naturel ». Nous avions l'impression

34 Le manuel d'usage actuel du N.Y. Times souligne toujours que le genre est principalement une référence grammaticale, bien qu'il admette les nouveaux sens que le terme a acquis dans le contexte social et politique ainsi que dans les idiomes comme «l'inégalité de genre » [NdTR «gender gap »]. De plus, le genre peut s'utiliser "pour éviter la confusion avec le sexe physique ou les doubles sens ». Mais l'expérience de certains contributeurs du N.Y. Times montre que la rédaction préfère sexe à genre à chaque fois que le corps est le sujet de discussion. 
enivrante de traiter de notre propre situation en révélant «le genre » comme une relation de pouvoir. De ce point de vue, nous pratiquions dans nos analyses du passé ce que Michel Foucault appelait « l'histoire du présent ». Et, en effet, nous avons produit beaucoup de travaux empiriques portant sur une grande variété de pratiques sociales et culturelles. Même les tensions entre féministes sur l'historicisation ou non des catégories «hommes» et «femmes» ont été, momentanément, productives dans la mesure où elles nous permettaient de débattre de l'implication politique de notre projet d'historicisation radicale. La tentative de Denise Riley de produire une généalogie foucaldienne des «femmes» sapait-elle inévitablement les fondements du projet politique féministe (comme Tania Modleski l'argumente dans Feminism Without Women), ou bien portait-elle notre critique de l'essentialisme jusqu'à sa conclusion ultime ? ${ }^{35} \mathrm{Y}$ avait-il une femme-sujet stable dont on pouvait raconter l'histoire malgré les différents contextes dans lesquels elle vivait? Étions-nous en train de produire ce sujet par le biais de notre recherche et de notre écriture, ou bien pré-existait-il à notre intérêt pour lui ? Quand le genre était compris comme une question ouverte sur les façons de concevoir la différence sexuelle, il servait de nouvelle catégorie d'analyse sociale, culturelle et historique. De surcroît, le genre a permis aux féministes de participer à un discours constructionniste plus large qui sapait la domination du structuralisme et du fonctionnalisme dans les sciences sociales et biologiques.

Je ne crois pas qu'il continue d'opérer ainsi, du moins pas aux États-Unis. Là, la prédominance toujours plus grande de la neurobiologie, de la microbiologie et des technologies de l'information, le grand intérêt pour le projet du génome humain ainsi que la recherche d'explications génétiques pour toutes les situations sociales et physiques ont sérieusement remis en question le constructionnisme, au moins ses notions trop simples comme celle de dire que cette science est seulement «sociale» et qu'on peut par conséquent ignorer son contenu. Alors que la discussion s'étend au sexe biologique (y-a-t-il un gène de l'homosexualité ? Les hormones féminines et la chirurgie de réparation rendent-elle les hommes qui

35 Riley 1988 ; Modleski 1991. 
ont été autrefois agressifs plus sympathiques, comme le soutient Donald/Deirdre McCloskey ? ${ }^{36}$ Les maladies sont-elles liées au sexe des personnes ?) et que le corps étend son emprise causale, les arguments constructionnistes, du moins ceux que nous utilisions autrefois pour insister sur les origines majoritairement sociales et étroitement politiques du savoir scientifique, sont devenus visiblement peu efficaces.

Peut-être le genre peut-il encore fournir une entrée critique dans les pays où le féminisme a peu pénétré le monde politique et universitaire, particulièrement là où il n'y a pas de traduction exacte pour ce mot? Mot importé de l'étranger, souvent non traduit, il sert de point de contestation pour toute une gamme de questions (sur la causalité biologique et culturelle dans le champ de la différence sexuelle autant que sur l'influence théorique du monde occidental), des questions posées par les féministes entre elles, entre elles et leurs alliés, et/ou leurs ennemis ${ }^{37}$. Mais, le genre a généralement atteint ce degré de neutralité scientifique et sociale qui le distingue de l'engagement qu'est le féminisme politique, lui garantissant par là son intégration universitaire. Le véritable enjeu aujourd'hui est la façon de prendre (ou non) position sur la question du féminisme. Le genre peut aussi bien être une façon de dissocier son travail des demandes spécifiques associées au féminisme, qu'il peut servir à déguiser les objectifs féministes des projets de recherche; dans les deux cas, l'enjeu est le féminisme et non «le genre ». Alors même que «les études de genre» prolifèrent dans de nombreux pays, notamment ceux de l'ancien bloc soviétique, il me semble évident que «le féminisme» a remplacé «le genre » comme motif de controverse internationale. Le féminisme est-il une importation occidentale ? Estil un mouvement international? Qui sont les femmes qui le peuplent? Le féminisme est-il un phénomène global ou local ? Qui

\footnotetext{
36 McCloskey 1999.

37 On me dit que le terme hébreu utilisé pour genre aujourd'hui en Israël est migdar. D'après l'anthropologue Moshe Shokeid, ce mot «a des résonances similaires à celles du terme anglais et sa racine se trouve très certainement dans gader, mur de séparation, clôture ou cloison ", c'est-à-dire une limite construite par les humains. Je remercie M. Shokeid pour cette référence.
} 
parle au nom des femmes? Ces questions nourrissent aujourd'hui un débat animé au sein de nombreux pays et au-delà des frontières des États-nations, des mouvements religieux et des organisations humanitaires. Le genre ne produit pas de controverse comparable ${ }^{38}$.

Le débat autour d'une politique féministe internationale fournit une illustration supplémentaire de l'efficacité passée et de l'inefficacité actuelle du genre comme catégorie d'intervention critique dans le débat politique et universitaire. Le "genre» était un terme controversé à la quatrième conférence internationale sur les femmes de l'Organisation des Nations Unies (ONU) à Pékin à l'automne 1995. Dans les semaines précédant la Conférence, une sous-commission de la Chambre des représentants étatsunienne a tenu des auditions au cours desquelles les élus républicains du Congrès et les groupes antiavortement ont détaillé les implications subversives du "genre »; ils ont instamment demandé au Congrès de ne pas financer la délégation officielle conduite par la Première dame Hillary Rodham Clinton. La morale et les valeurs familiales, disaient leurs porte-parole, étaient attaquées par ceux qui pensaient qu'il pouvait y avoir jusqu'à cinq genres (hommes, femmes, homosexuels, bisexuels et transsexuels). Le programme de l'ONU pour la conférence de Pékin, ont-ils ajouté, avait été détourné par

les féministes du genre, qui croient que tout ce que nous pensons comme naturel dont les hommes, les femmes, la féminité et la masculinité, la maternité et la paternité, l'hétérosexualité, le mariage et la famille ne sont que des arrangements d'origine culturelle, créés par les hommes pour opprimer les femmes. Ces féministes professent que ces rôles sont des constructions sociales, donc assujetties au changement ${ }^{39}$.

$\mathrm{Au}$ sein de l'ONU, la controverse était telle que la commission sur le statut des femmes avait établi un groupe pour rechercher un accord sur «le sens généralement admis de 'genre'» et pour communiquer ses conclusions «directement au colloque de Pékin ». Le désaccord

38 Pour se faire une idée de cette controverse, voir Scott, Kaplan \& Keates 1997.

39 U.S. House of Representatives, 104th Congress, First Session, Committee on International Relations: United Nations Fourth World Conference on Women Hearings before the Subcommittee on International Operations and Human Rights, 18 juillet, 2 août 1995, Washington D.C., U.S. Government Printing Office, 1996, p. 43. 
entre les partisans d'une définition strictement biologique et ceux qui voulaient faire référence "aux rôles socialement constructifs [sic] des hommes et des femmes $»^{40}$ a abouti à une résolution qui n'apportait aucune information, mais a néanmoins été ajoutée en annexe au programme d'actions de la conférence. La «Déclaration sur le sens communément admis du terme 'genre'» est la suivante :

Ayant longuement réfléchi à cette question, le groupe a remarqué que (1) le terme "genre» avait déjà été communément utilisé et compris dans son usage généralement admis dans de nombreux autres forums et colloques de l'ONU ; (2) il n'y avait aucune indication qu'un nouveau sens ou nouvelle connotation du terme, différent du sens précédemment en usage, soit intentionnel dans le programme d'action [...]. De sorte que le groupe a réaffirmé que le terme «genre » tel qu'il était utilisé dans le programme d'action était à interpréter et comprendre selon son usage courant et généralement admis ${ }^{41}$.

Ce qui est frappant dans cette tentative de clarification est qu'il n'existe nulle part d'explication sur cet «usage courant et généralement admis». C'était comme si le sens allait de soi, était dégagé de toute ambigüité et de toute mauvaise interprétation. La formulation de la déclaration, bien sûr, essaie de supprimer la controverse en niant son existence. Pourtant, certaines participantes à la Conférence se sont senties obligées de clarifier leur compréhension du terme. La représentante du Guatemala, par exemple, écrit que

conformément aux critères éthique, moral, légal, culturel et naturel du peuple guatémaltèque, le Guatemala interprète le concept de genre exclusivement dans le sens du genre féminin et du genre masculin en référence aux femmes et aux hommes ${ }^{42}$.

$40 \quad$ Ibid., p. 107.

41 United Nations Commission on the Status of Women, Report of the United Nations Conference on Human Settlements (Habitat II), Istanbul, 3-14 June 1996, Annex V: Statement on the Commonly Understood Meaning of the Term 'Gender'.

Voir http://ww2.unhabitat.org/unchs/english/hagenda/annex5.htm.

42 United Nations, Report of the Fourth World Conference on Women, Beijing, 415 September 1995, chapter V, section 10 (b), iii.

Voir http://www.un.org/esa/gopher-data/conf/fwcw/off/a--20.en. 
Le Paraguay a fait une déclaration similaire. Le Pérou est allé plus loin, anticipant les implications dangereuses que «le genre» semble avoir en insistant sur le fait que « les droits sexuels ne concernent que les relations hétérosexuelles $»^{43}$. Enfin, le représentant du Vatican a interprété le sens commun de "genre » comme « ancré dans l'identité sexuelle biologique, qu'elle soit masculine ou féminine ». "Le SaintSiège exclut ainsi les interprétations douteuses fondées sur des représentations selon lesquelles l'identité sexuelle peut s'adapter indéfiniment à des objectifs différents et renouvelés ». Non pas que la biologie déterminât les rôles sexués de façon statique. Le Pape était tout à fait favorable à « une certaine diversité de rôles [...] à condition que cette diversité ne soit pas le résultat d'une position arbitraire, mais l'expression de ce qui est spécifique à l'homme et à la femme $»^{44}$ (en d'autres termes, l'homosexualité n'était pas de l'ordre de la nature et ne pouvait pas être concernée par "le genre »).

D'un côté, cette avalanche d'objections témoigne du potentiel radical du «genre » pour dénaturaliser le sexe en le relativisant et l'historicisant (ce qui contredit mes affirmations précédentes sur les limites de l'analyse par le genre). Les porte-parole catholiques ont clairement identifié le danger menaçant le dogme religieux : si le sexe est un effet du genre, il n'y a pas de corrélation obligée entre le corps physique, le rôle social et le comportement sexuel. Dans les jours qui ont précédé Pékin, le Pape a nommément mentionné parmi les théoriciennes développant cet argument, Judith Butler, pour son plus grand bonheur et celui de ses amies et admiratrices. D'un autre côté, les féministes peuvent ridiculiser le fantasme des cinq genres, mais elles ne peuvent pas réfuter catégoriquement l'affirmation que le genre humain est seulement constitué d'hommes et de femmes. Encore plus révélateur, peut-être, le rapport final de Pékin et la déclaration sur «l'usage courant» montrent que l'inquiétude de Rome n'était pas fondée. Comme l'entrée «genre» de l'American Heritage Dictionary l'indique, le genre est devenu simplement une autre façon de faire référence aux femmes et aux hommes. Dans le rapport de Pékin, «l'égalité de genre » signifie l'égalité entre les femmes et les

\footnotetext{
43 Ibid., section 25, iii.

44 Ibid., section II, «Statement of interpretation of the term 'gender' ».
} 
hommes, «l'équilibre de genre » est une juste représentation pour les deux sexes ; "la conscience de genre », qui est censée nourrir toutes les prises de décisions, aborde la façon dont les politiques appliquées peuvent affecter différemment les hommes et les femmes. L'assemblée générale a demandé aux gouvernements et aux Organisations non gouvernementales (ONG) de «maintenir une perspective de genre dans toutes les politiques et programmes, de telle sorte qu'avant de prendre une décision, on analyse les effets respectifs sur les femmes et les hommes $»^{45}$. Cela signifie, en gros, que les statistiques et les prospectives doivent êtres calculées par sexe. Les 200 mentions environ de "genre» dans le programme d'actions de Pékin remplacent souvent simplement le mot «femmes». "Le genre » indique aussi, et c'est important, qu'on croit à la possibilité de modifier le rôle des femmes pour le meilleur et d'atteindre une certaine égalité entre les sexes. C'est une façon discrète d'approuver certains aspects du programme féministe pour l'égalité.

Pourtant, l'usage du terme "genre», même s'il signale une ouverture au changement concernant certains rôles traditionnels des femmes et des hommes, n'a aucune des qualités subversives qui effrayent tant ses critiques. Dans l'usage courant, "le genre » est devenu un synonyme des différences entre les sexes, à la fois attribuables et «naturelles». Je pense que cette limitation des possibilités subversives du genre est partiellement le résultat du triomphe des forces conservatrices à Pékin (et ailleurs). C'est aussi le résultat de la distinction sexe/genre elle-même, qui tend implicitement à confirmer le discours biologique soulignant le caractère anhistorique du corps physique (et qui ne conteste pas l'essentialisme qui voudrait que le sexe détermine le genre). C'est une limitation cruciale à un moment où il y a confluence d'un large éventail de facteurs, de l'influence importante du mouvement gay et lesbien à la prééminence de la théorie évolutionniste dans le champ des études cognitives et de la microbiologie : le sexe et la différence sexuelle sont au cœur d'un débat politique et scientifique animé.

Alors que les années 1990 se terminent, les limites du genre me semblent toujours plus évidentes. Aux États-Unis (et à l'ONU), le

45 Ibid., chapitre IV, D, section 123. 
genre est devenu d'un usage courant, communément proposé comme un synonyme de femmes, de différences entre les sexes, de sexe. Parfois, ce terme dénote les règles sociales imposées aux femmes et aux hommes, mais il ne se réfère quasiment jamais au savoir qui organise notre perception de la nature. Les ouvrages qui se proposent d'offrir une «analyse du genre » sont généralement des études tout à fait prévisibles sur les femmes ou (comme le programme d'actions de Pékin) un examen des différences de statut, d'expérience et de possibilités ouvertes aux femmes et aux hommes. Ces ouvrages examinent rarement comment le discours établit le sens de «femmes» et $\mathrm{d}^{\prime}$ '«hommes », quelles contradictions le traversent, ce que ces termes excluent, quelles sont les variations d'expériences vécues par les «femmes» dans les différents régimes normatifs du genre, quelles sont les relations, si elles existent, entre la compréhension scientifique actuelle, disons, des processus cognitifs ou de l'évolution de l'espèce et celle de la différence des sexes. Il est vrai que certaines chercheuses féministes utilisent le terme genre alors qu'elles rejettent explicitement le postulat que «hommes» et «femmes» sont des catégories historiques variables. Ceci a eu pour effet de réifier l'opposition homme/femme comme fondatrice et fondamentale (c'est-à-dire d'accepter les termes de la psychologie évolutionniste) et donc de nier le potentiel radical du genre tant dans le domaine de la recherche que dans le champ politique.

C'est ainsi que je me retrouve à utiliser de moins en moins le terme genre dans mon travail et à préférer parler de la différence des sexes (terme qui ne présume pas de différences fixes, mais étudie leur mode opératoire) et du sexe biologique en tant que variables conceptuelles et historiques. Tout ceci ne résout pas le problème que j'ai décrit ici, puisque le risque est grand (surtout dans le contexte discursif actuel) qu'on comprenne ceci comme l'acceptation idéologique que le sexe est un fait de la nature ${ }^{46}$. Malgré tout, il me

46 Il serait instructif de comparer avec les efforts insistant sur la construction sociale de la race. Ceux qui veulent contrer les arguments essentialistes liés à la race n'ont pas eu à développer une distinction similaire à celle du sexe et du genre parce que l'idée de la race comme fait naturel avait été discréditée par les 
semble nécessaire de regarder ailleurs pour trouver des termes et des théories qui vont rompre la routine habituelle de l'histoire en général et de l'histoire des femmes en particulier. Je ne suis pas en train d'écrire que nous devons éliminer de notre vocabulaire le genre et les notions qui lui sont associées. Je ne pense pas non plus qu'il faille faire la chasse aux mauvais usages du terme au profit du seul nôtre. Ceci serait une tâche non seulement impossible, mais aussi absurde car elle nierait la flexibilité et la mobilité du langage, son rôle crucial comme acteur du changement. Je pense que nous devons avancer dans une nouvelle direction et nous dégager de nos présupposés routiniers. C'est précisément quand nous savons ce qu'un terme veut dire, quand son usage est si communément admis que son sens ne nécessite plus d'être combattu ni promu, que nous devons chercher à redéfinir les mots et concepts, ou bien à redéployer et reformuler les idées existantes.

La raison d'être du questionnement féministe, et pour moi son intérêt durable, tient à son refus de maintenir le statu quo. Le féminisme a toujours résisté à la consolidation du terme "femmes » en une catégorie homogène, même lorsqu'il a lancé des appels politiques en leur nom à toutes. Bien que cette tension ait perturbé celles qui cherchaient la sécurité qu'apporte une identité fixe, elle a également été à l'origine des campagnes politiques les plus créatives du féminisme. Au sommet de son efficacité, le féminisme crée la consternation en pointant les contradictions et les incohérences de sociétés pourtant favorables à l'égalité et à la justice pour tous. En effet, parce que les revendications des féministes ont surpris le conformisme dominant, parce qu'elles ont refusé ou reformulé «les usages communément admis », elles ont pu attirer l'attention sur leur cause $^{47}$. Comme le genre est devenu un mot associé à une certaine orthodoxie féministe ainsi qu'à "l'usage courant», il est temps de réfléchir à ses limites, temps de chercher de nouvelles conceptualisations du problème du sexe et de la différence sexuelle qui permettront au questionnement féministe de se tonifier, tout en

anthropologues dans les années 1920, puis politiquement après la Seconde Guerre mondiale. Sur la race, voir Roberts 1995 ; Proctor 1997 ; Stocking 1982.

47 Scott 1998. 
continuant à jouer son rôle traditionnellement provocateur et perturbateur.

\section{Traduit de l'américain par Myriam Boussabba-Bravard}

\section{Bibliographie}

Browne Kingsley, 1999, Divided Labors: an Evolutionary View of Women at Work, New Haven, Yale University Press.

Butler Judith, 2009 [éd. américaine 1993], Ces corps qui comptent. De la matérialité et des limites discursives $d u$ "sexe", Paris, Éditions Amsterdam, traduit de l'américain par Charlotte Nordmann.

CONWAY Jill, 1972, "Stereotypes of Femininity in a Theory of Evolution », in Martha VICINUS (ed.), Suffer and Be Still: Women in the Victorian Age, Bloomington, Indiana University Press, 1972, p. 140-154.

DAwSON Doyne, 1999, «Evolutionary Theory and Group Selection: the Question of Warfare », History and Theory, 38/4, p. 79-100.

DeAcon Terrence W., 1997, The Symbolic Species: the Co-evolution of Language and the Brain, New York, W.W. Norton.

DupRÉ John, 1993a, The Disorder of Things: Metaphysical Foundations of the Disunity of Science, Cambridge Mass., Harvard University Press.

—, 1993b, "Scientism, Sexism, and Sociobiology: one More Link in the Chain ", Behavioral and Brain Sciences, 16/2, p. 292.

—, 1999, compte rendu de Steven Pinker, How the Mind Works in Philosophy of Science, 66/3, p. 489-493.

Emilio John D' \& Estelle B. Freedman, 1988, Intimate Matters: a History of Sexuality in America, New York, Harper \& Row.

FISHER Helen, 1999, The First Sex: the Natural Talents of Women and how they are changing the World, New York, Random House.

Foucault Michel, 1976, Histoire de la sexualité, I. La volonté de savoir, Paris, Gallimard.

Fracchia Joseph \& Richard LEWONTIN, 1999, « Does Culture Evolve? », History and Theory, 38/4, p. 52-75.

FREUD Sigmund, 1962, Trois essais sur la théorie de la sexualité, trad. B. Reverchon-Juve, Paris, Gallimard.

GEERTZ Clifford, 1973, The Interpretation of Cultures: Selected Essays, New York, Basic Books. 
Gould Stephen Jay, 1997, «Evolution: the Pleasures of Pluralism », New York Review of Books, 26 juin.

Grosz Elizabeth, 1994, «Experimental Desire: Rethinking Queer Subjectivity », in Joan Copjec (ed.), Supposing the Subject, Londres, Verso, p. 133-157.

Haraway Donna, 1991, Simians, Cyborgs and Women: the Reinvention of Nature, New York, Routledge.

Lewontin Richard, Rose Steven \& Leon J. Kamin, 1984, Not in Our Genes: Biology, Ideology, and Human Nature, New York, Pantheon books.

MaLik Kenan, 1998, «Darwinian Fallacies », Prospect, décembre, p. 24-30.

McCloskey Deirdre, 1999, Crossing: A Memoir, Chicago, Chicago University Press.

Miller Jacques-Alain (ed.), 1978, LACAN Jacques, The Four Fundamental Concepts of Psycho-Analysis, trad. Alan Sheridan, New York, Norton.

Modleski Tania, 1991, Feminism Without Women: Culture and Criticism in a Post-Feminist Age, New York, Routledge.

OrTneR Sherry \& Harriet WhiteHead (eds), 1991, Sexual Meanings: the Cultural Construction of Gender and Sexuality, Cambridge, Cambridge University Press.

PINKER Steven, 1988, « Boys will be Boys », The New Yorker, 9 février.

Proctor Robert N., 1997, "Race since Boas: The Shadow of Fascism and the Challenge of Molecular Anthropology », communication non publiée, novembre.

Riley Denise, 1988, 'Am I that Name?' Feminism and the Category of 'Women' in History, Houndsmills, Macmillan Press.

RoBerTs Dorothy E., 1995, "The Genetic Tie », The University of Chicago Law Review, 62/1, p. 209-273.

Rosaldo Michelle Z., 1980, "The Use and Abuse of Anthropology: Reflections on Feminism and Cross-Cultural Understanding », Signs, 5/3, p. 389-417.

Rubin Gayle, 1975, "The Traffic in Women: Notes on the 'Political Economy' of Sex », in Rayna R. ReITER (ed.), Toward an Antbropology of Women, New York, Monthly Review Press, p. 157-210.

SCHWARTZ James, 1999, «Oh, My Darwin! Who's the Fittest Evolutionary Thinker of Them All? », Lingua Franca, 9/8.

SCOTT Joan W., KAPLAN Cora \& Debra KEATES (eds), 1997, Transitions, Environments, Translations: Feminism in International Politics, New York, Routledge.

SCOTT Joan W., 1998 [éd. américaine 1996], La citoyenne paradoxale. Les féministes français et les droits de l'homme, Paris, Albin Michel.

SHAw David Gary, 1999, "The Return of Science », History and Theory, 38/4, p. 1-9. 
ShePherdson Charles, 2000, Vital Signs: Nature, Culture, Psychoanalysis, New York, Routledge.

STERLING Anne Fausto, 1985, Myths of Gender: Biological Theories about Women and Men, New York, Basic Books.

-, 2000, Sexing the Body: Gender Politics and the Construction of Sexuality, New York, Basic Books.

STOCKING George W. Jr., 1982 [1 1re éd. 1968], Race, Culture and Evolution: Essays in the History of Anthropology, Chicago, Chicago University Press.

WiLSON Elizabeth A., 1998, Neural Geographies: Feminism and the Microstructure of Cognition, New York, Routledge.

ŽIŽEK Slavoj, 1997, The Plague of Fantasies, Londres, Verso. 